\title{
LANDAU-LIFSHITZ EQUATION OF FERROMAGNETISM WITH EXTERNAL MAGNETIC FIELD
}

\author{
P. Y. H. PANG, J. XIAO and F. ZHOU
}

(Received 10 April 2000; revised 26 February 2001)

Communicated by $\mathrm{K}$. Wysocki

\begin{abstract}
In this article, we prove the existence and uniqueness of solution for the Cauchy problem of the LandauLifshitz equation of ferromagnetism with external magnetic field. We also show that the solution is globally regular with the exception of at most finitely many blow-up points. An energy identity at blow-up points is presented.
\end{abstract}

2000 Mathematics subject classification: primary 35Q60, 35B65, 82D40.

Keywords and phrases: Landau-Lifshitz equation, regularity, energy identity.

\section{Introduction}

In this paper we discuss the Landau-Lifshitz equation [10] which models the ferromagnetic spin chain on surfaces with the Gilbert damping term in the presence of an external magnetic field $h(x, t)$. This equation plays an important rôle in the understanding of non-equilibrium magnetism.

Let $\mathscr{M}$ and $\mathscr{N}$ be closed oriented Riemannian surfaces with metric tensors $\gamma=$ $\left(\gamma_{\alpha \beta}\right)_{1 \leq \alpha, \beta \leq 2}$ and $g=\left(g_{i j}\right)_{1 \leq i, j \leq 2}$ respectively. Let $\mathscr{N}$ be isometrically embedded in $\mathbb{R}^{3}$ and denote its second fundamental form by $A$. We shall assume that the principal curvatures of $\mathscr{N}$ and their derivatives are uniformly bounded. Throughout this paper, $C$ will denote constants which depend only on $\mathscr{M}$ and $\mathscr{N}$. The notation ' $\because$ for the dot product of vectors will be suppressed.

For $u_{0}: \mathscr{M} \rightarrow \mathscr{N}$ and $t>0$, the Landau-Lifshitz system is given by

$$
\partial_{\imath} u=-\alpha_{1} \phi(u) \times(\phi(u) \times(\Delta u+h))+\alpha_{2} \phi(u) \times(\Delta u+h), \quad u(\cdot, 0)=u_{0},
$$

(C) 2002 Australian Mathematical Society $1446-7887 / 2000 \$ A 2.00+0.00$ 
where $\alpha_{1}>0$ and $\alpha_{2}$ are constants, $\times$ denotes the vector cross product in $\mathbb{R}^{3}, \Delta$ denotes the Laplace-Beltrami operator on $\mathscr{M}, \phi$ is a vector field in $\mathbb{R}^{3}$, which restricts to a unit normal vector field on $\mathscr{N}$, and $h(x, t)=\left(h_{1}(x, t), h_{2}(x, t), h_{3}(x, t)\right)$ represents the external magnetic field with $h_{0}=h(\cdot, 0)$. Furthermore, we shall assume that

$$
\begin{gathered}
u_{0} \in W^{1,2}(\mathscr{M}, \mathscr{N}), \quad h_{0} \in L^{2}(\mathscr{M}, \mathscr{N}) ; \quad H_{\infty}=\underset{(x, t) \in \mathscr{M}^{\infty}}{\operatorname{ess} \sup }|h(x, t)|<\infty ; \\
H_{0}=\int_{\mathscr{M}^{\infty}}|h(x, t)|^{2}<\infty ; \quad H_{1}=\int_{\mathscr{M}^{\infty}}\left|\partial_{t} h(x, t)\right|^{2}<\infty,
\end{gathered}
$$

where the notation $\mathscr{M}_{s}^{T}:=\mathscr{M} \times[s, T] \subset \mathscr{M} \times[0, \infty]$ is used. In the case $s=0$, we just write $\mathscr{M}^{T}$.

For $\Omega \subset \mathscr{M}$, the energy of the map $u: \Omega \rightarrow \mathscr{N}$ is given by

$$
E_{\Omega}(u)=\int_{\Omega} e(u) d \mathscr{M}
$$

in terms of the energy density $e(u)$, which is defined in local coordinates by

$$
e(u)=\frac{1}{2}|\nabla u|^{2}=\frac{1}{2} \gamma^{\alpha \beta}(x) g_{i j}(u) \frac{\partial}{\partial x^{\alpha}} u^{i} \frac{\partial}{\partial x^{\beta}} u^{j},
$$

where $\left(\gamma^{\alpha \beta}\right)=\left(\gamma_{\alpha \beta}\right)^{-1}$. For $\Omega=B_{R}(x)$, the geodesic ball of radius $R$ centred at $x$, we also write $E_{\Omega}(u)=E_{R}(u ; x)$.

In the absence of the external magnetic field, that is, $h=0$, equation (1.1) was discussed in [14]. In addition, if the target manifold is $S^{2}$, the equation was discussed in detail in [6], see also [4]. In this case, with the presence of an external magnetic field but without the Gilbert damping term, that is, $\alpha_{2}=0,(1.1)$ becomes $\partial_{t} u=$ $\Delta u+|\nabla u|^{2} u+h-(h, u)$. Its static form $\Delta u+|\nabla u|^{2} u+h-(h, u)=0$ was discussed in several papers, see for example $[8,9]$. If $\alpha_{1}=0$, the problem was studied in [17].

As $\phi(u) \times(\phi(u) \times \Delta u)=(\phi(u) \Delta u) \phi(u)-|\phi(u)|^{2} \Delta u,|\phi(u)|^{2}=1$, "and $(\phi(u) \Delta u) \phi(u)=(\Delta u)^{N}=-A(u)(\nabla u, \nabla u)$, we note that the equation (1.1) has the following equivalent form in terms of the second fundamental form $A$ of $\mathscr{N}$ :

$$
\begin{aligned}
\partial_{t} u= & \alpha_{1} \Delta u+\alpha_{1} A(u)(\nabla u, \nabla u) \\
& -\alpha_{1} \phi(u) \times(\phi(u) \times h)+\alpha_{2} \phi(u) \times(\Delta u+h) .
\end{aligned}
$$

Note that taking only the first two terms on the right-hand side of (1.3) gives us the evolution equation of harmonic maps. The equation $\partial_{t} u=\Delta u+A(u)(\nabla u, \nabla u)$ was fully discussed in [15]. If the initial data $u_{0} \in W^{1,2}(\mathscr{M}, \mathscr{N})$ is given, its unique solution exists and is smooth globally except at finitely many blow-up points. Energy identities describing the solutions near the blow-up points were given in a number of 
papers, for example, [3-5, 11-13]. Examples of finite time blow-up for the harmonic map heat flow were constructed in $[1,2]$.

In this paper, we discuss the existence, uniqueness and regularity of solutions to (1.1). Our results are as follows:

THEOREM 1 (Existence and Uniqueness). For any initial value $u_{0}$ and the external magnetic field $h(x, t)$ satisfying (1.2), there exists a unique solution $u$ of (1.1) which is regular on $\mathscr{M} \times(0, \infty)$ with the exception of at most finitely many points $\left(x^{l}, T^{l}\right)$, $1 \leq l \leq L$. Furthermore, the singular points are characterized by the condition that

$$
\limsup E_{R}\left(u(\cdot, t) ; x^{l}\right)>\varepsilon_{0} \quad \text { for all } R \in\left(0, R_{0}\right]
$$
$\rightarrow T^{t^{-}}$

where $\varepsilon_{0}=\varepsilon_{0}(\mathscr{M}, \mathscr{N})$ and $R_{0}=R_{0}(\mathscr{M})$ are positive constants (to be specified later).

The solution obtained in Theorem 1 is called 'almost smooth'.

THEOREM 2 (Energy Identity). For an almost smooth solution $u$ of (1.1), let $S$ denote the set of singularities, and we suppose $\left(x_{0}, T\right) \in S$ where $0<T \leq \infty$. Then, there exist

(i) a sequence $t_{n} \rightarrow T$,

(ii) harmonic maps $\omega_{i}: S^{2} \rightarrow \mathscr{N}, 1 \leq i \leq m<\infty$,

(iii) $\left\{a_{n}^{i}\right\}_{i=1}^{m} \subset \mathscr{M}$ and $\left\{\lambda_{n}^{i}\right\}_{i=1}^{m} \subset \mathbb{R}^{+}$, with $\lim _{n \rightarrow \infty} a_{n}^{i}=x_{0}, \lim _{n \rightarrow \infty} \lambda_{n}^{i}=0$

and

$$
\lim _{n \rightarrow \infty} \frac{\lambda_{n}^{i}}{\lambda_{n}^{j}}+\frac{\lambda_{n}^{j}}{\lambda_{n}^{i}}+\frac{\left|a_{n}^{i}-a_{n}^{j}\right|^{2}}{\lambda_{n}^{j} \lambda_{n}^{i}}=\infty, \quad j \neq i
$$

such that

$$
u\left(x, t_{n}\right)-\sum_{i=1}^{m}\left(\omega_{i}\left(\frac{x-a_{n}^{i}}{\lambda_{n}^{i}}\right)-\omega_{i}(\infty)\right) \rightarrow u(x, T)
$$

strongly in $W^{1,2}\left(B_{R}\left(x_{0}\right), \mathscr{N}\right)$ for any disk $B_{R}\left(x_{0}\right)$ with $\overline{B_{2 R}\left(x_{0}\right)} \bigcap\left\{x \neq x_{0}:(x, T) \in\right.$ $S\}=\emptyset$. Here, $u(\cdot, T)$ denotes the weak limit of $u\left(\cdot, t_{n}\right)$, and $\omega_{k}(\infty)$ refers to the image under $\omega_{k}$ of the north pole of $S^{2}$. Furthermore, we have the following energy identity:

$$
\lim _{n \rightarrow \infty} E_{R}\left(u\left(\cdot, t_{n}\right) ; x_{0}\right)=E_{R}\left(u(\cdot, T) ; x_{0}\right)+\sum_{i=1}^{m} E\left(\omega_{i}\right) .
$$

REMARK 1. In Theorem 2, if $T=\infty$, we emphasize that $t_{n}$ has to be chosen appropriately in order for the pointwise weak limit $u(x, \infty)$ to be a harmonic map. On the other hand, if $T<\infty$, the energy identity (1.7) is independent of the choice of the sequence $\left\{t_{n}\right\}$ once the bubbles $\left\{\omega_{i}\right\}_{i=1}^{m}$ have been selected. 


\section{Existence and uniqueness}

The proof of Theorem 1 is given in this section. First, following [15], we define the following function space:

$$
\begin{aligned}
V\left(\mathscr{M}_{s}^{T} ; \mathscr{N}\right):= & \{u: \mathscr{M} \times[s, T] \rightarrow \mathscr{N} \mid u \text { measurable, } \\
& \left.\quad \underset{s \leq t \leq T}{\operatorname{essup}} \int_{\mathscr{M}}|\nabla u(\cdot, t)|^{2}+\int_{s}^{T} \int_{\mathscr{M}}\left(\left|\nabla^{2} u\right|^{2}+\left|\partial_{t} u\right|^{2}\right)<\infty\right\} .
\end{aligned}
$$

REMARK 2. There exist constants $C$ and $R_{0}>0$ such that for any $u \in V\left(\mathscr{M}^{T} ; \mathscr{N}\right)$ and any $R \in\left(0, R_{0}\right]$ we have

$$
\int_{\mathscr{M}^{T}}|\nabla u|^{4} \leq C \operatorname{ess}_{(x, t) \in \mathscr{M}^{T}} E_{R}(u(\cdot, t) ; x)\left(\int_{\mathscr{M}^{T}}\left|\nabla^{2} u\right|^{2}+R^{-2} \int_{\mathscr{N}^{T}}|\nabla u|^{2}\right),
$$

where $R_{0}=R_{0}(\mathscr{M})$ is a constant which arises from the covering lemma [15, Lemma 3.3] (see also [15, Lemma 3.1]).

LEMMA 1. Let $u$ be a solution of (1.1), then

$$
\alpha_{2} \int_{\mathscr{M}^{T}} \partial_{t} u(\phi(u) \times(\Delta u+h))=\frac{\alpha_{2}^{2}}{\alpha_{1}^{2}+\alpha_{2}^{2}} \int_{\mathscr{M}^{T}}\left|\partial_{t} u\right|^{2} .
$$

PROOF. Multiplying (1.1) by $\phi(u)$ and observing that $-\phi(u) \times(\phi(u) \times(\phi(u) \times$ $(\Delta u+h)))=\phi(u) \times(\Delta u+h)$, we get

$$
\phi(u) \times \partial_{t} u=\alpha_{1} \phi(u) \times(\Delta u+h)+\alpha_{2} \phi(u) \times(\phi(u) \times(\Delta u+h)) .
$$

Using (1.1) again, we get

$$
\phi(u) \times \partial_{t} u+\frac{\alpha_{2}}{\alpha_{1}} \partial_{t} u=\frac{\alpha_{1}^{2}+\alpha_{2}^{2}}{\alpha_{1}}(\phi(u) \times(\Delta u+h)) .
$$

The result (2.2) now follows by multiplying both sides of (2.3) by $\partial_{\imath} u$ and using the fact that $\partial_{t} u\left(\phi(u) \times \partial_{t} u\right)=0$.

REMARK 3. Similarly, it can be proved that for any $\varphi \in C_{0}^{\infty}\left(B_{2 R}(x)\right)$ satisfying $0 \leq \varphi \leq 1, \varphi \equiv 1$ on $B_{R}(x)$, and $|\nabla \varphi| \leq C / R$ for some constant $C$, we have

$$
\alpha_{2} \int_{\mathscr{M}^{T}} \partial_{t} u(\phi(u) \times(\Delta u+h)) \varphi^{2}=\frac{\alpha_{2}^{2}}{\alpha_{1}^{2}+\alpha_{2}^{2}} \int_{\mathscr{M}^{T}}\left|\partial_{t} u\right|^{2} \varphi^{2} .
$$


LEMMA 2. Let $u \in V\left(\mathscr{M}^{T} ; \mathscr{N}\right)$ be a solution of $(1.1)$, then there exists a constant C such that

$$
\int_{\mathscr{K}^{T}}\left|\partial_{t} u\right|^{2} \leq C\left(E_{0}+H_{0}\right)
$$

where $E_{0}=\int_{\mathscr{M}}\left|\nabla u_{0}\right|^{2}$. Moreover, $E(\cdot, t)$ is absolutely continuous and uniformly bounded on $[0, T]$ :

$$
E(\cdot, t) \leq E_{0}+C H_{0},
$$

and the tension field $\tau(u)=\Delta u+A(u)(\nabla u, \nabla u)$ satisfies

$$
\int_{\mathscr{M}^{T}}|\tau(u)|^{2} \leq C\left(E_{0}+H_{0}\right) .
$$

PROOF. Multiplying both sides of (1.3) by $\partial_{t} u$ and integrating, we obtain

$$
\begin{gathered}
\int_{\mathscr{K}_{s}^{\prime}}\left|\partial_{t} u\right|^{2}+\alpha_{1} \int_{s}^{t} \frac{d}{d t} E(u(\cdot, t)) d t+\alpha_{1} \int_{\mathscr{H}_{s}^{t}} \partial_{t} u(\phi(u) \times(\phi(u) \times h)) \\
-\alpha_{2} \int_{\mathscr{N}_{s}^{\prime}} \partial_{t} u(\phi(u) \times(\Delta u+h))=0 .
\end{gathered}
$$

By virtue of

$$
\int_{\mathscr{N}_{s}^{\prime}} \partial_{t} u(\phi(u) \times(\phi(u) \times h)) \leq \int_{\mathscr{M}_{s}^{\prime}}\left|\partial_{t} u\right||h| \leq \frac{\alpha_{1}}{2\left(\alpha_{1}^{2}+\alpha_{2}^{2}\right)} \int_{\mathscr{M}_{s}^{\prime}}\left|\partial_{t} u\right|^{2}+C \int_{\mathscr{M}_{s}^{\prime}}|h|^{2}
$$

and Lemma 1, we get

$$
\frac{\alpha_{1}}{2\left(\alpha_{1}^{2}+\alpha_{2}^{2}\right)} \int_{\mathscr{M}_{s}^{\prime}}\left|\partial_{t} u\right|^{2}+\int_{s}^{t} \frac{d}{d t} E(u(\cdot, t)) d t \leq C \int_{\mathscr{M}_{s}^{\prime}}|h|^{2} \leq C H_{0} .
$$

Inequalities (2.5) and (2.6) now follow directly from (2.8). For (2.7), we notice that the two terms on the right-hand side of (1.1) are orthogonal to each other, so

$$
\begin{aligned}
\alpha_{1}^{2} \int_{\mathscr{M}_{s}^{\prime}}|\tau(u)|^{2} & =\alpha_{1}^{2} \int_{\mathscr{M}_{s}^{\prime}}|\phi(u) \times(\phi(u) \times \Delta u)|^{2} \\
& \leq 2 \alpha_{1}^{2} \int_{\mathscr{M}_{s}^{\prime}}|\phi(u) \times(\phi(u) \times(\Delta u+h))|^{2}+2 \alpha_{1}^{2} \int_{\mathscr{M}_{s}^{\prime}}|h|^{2} \\
& =2 \int_{\mathscr{M}_{s}^{\prime}}\left|\partial_{t} u\right|^{2}-2 \alpha_{2}^{2} \int_{\mathscr{M}_{s}^{\prime}}|\phi(u) \times(\Delta u+h)|^{2}+2 \alpha_{1}^{2} \int_{\mathscr{M}_{s}^{\prime}}|h|^{2} \\
& \leq 2 \int_{\mathscr{M}_{s}^{\prime}}\left|\partial_{t} u\right|^{2}+2 \alpha_{1}^{2} \int_{\mathscr{N}_{s}^{\prime}}|h|^{2} \leq C\left(E_{0}+H_{0}\right) .
\end{aligned}
$$


REMARK 4. Combining (2.1) and (2.6) we obtain the estimate

$$
\int_{\mathscr{M}^{T}}|\nabla u|^{4} \leq C \underset{(x, t) \in \mathscr{M}^{T}}{\operatorname{ess} \sup _{R}} E_{R}(u(\cdot, t) ; x)\left(\int_{\mathscr{K}^{T}}\left|\nabla^{2} u\right|^{2}+\frac{T}{R^{2}}\left(E_{0}+H_{0}\right)\right)
$$

for any solution $\mathrm{u} \in V\left(\mathscr{M}^{T} ; \mathscr{N}\right)$ of $(1.1)$ and any $R \in\left(0, R_{0}\right]$.

Now we prove a lemma which provides a local estimate on the energy.

LEMMA 3. There exists a constant $C$ such that for any solution $u \in V\left(\mathscr{M}^{T} ; \mathscr{N}\right)$ of (1.1), and any $R \in\left(0, R_{0}\right]$, the estimate

$$
E_{R}(u(\cdot, t) ; x) \leq E_{2 R}\left(u_{0} ; x\right)+C \frac{t}{R^{2}}\left(E_{0}+H_{*}\right)
$$

holds. (Here $H_{*}=H_{*}(\mathscr{M}, h)$ is a constant.)

ProOF. The equation (1.3) is equivalent to

$$
\partial_{t} u-\alpha_{1} A(u)(\nabla u, \nabla u)-\alpha_{2} \phi(u) \times(\Delta u+h)=\alpha_{1} \Delta u-\alpha_{1} \phi(u) \times(\phi(u) \times h) \text {. }
$$

In view of

$$
\begin{aligned}
& \int_{\mathscr{M}_{s}^{\prime}}\left|\alpha_{1} \phi(u) \times(\phi(u) \times h) \partial_{t} u\right| \varphi^{2} \\
& \quad \leq \alpha_{1} \int_{\mathscr{N}_{j}^{\prime}}|h|\left|\partial_{t} u\right| \varphi^{2} \leq \frac{\alpha_{1}^{2}}{2\left(\alpha_{1}^{2}+\alpha_{2}^{2}\right)} \int_{\mathscr{M}_{s}^{\prime}}\left|\partial_{t} u\right|^{2} \varphi^{2}+C \int_{\mathscr{N}_{s}^{\prime}}|h|^{2} \varphi^{2}
\end{aligned}
$$

and Remark 3, we multiply (2.10) by $\left(\partial_{t} u\right) \varphi^{2}$, where $\varphi$ is as stated in Remark 3, and then integrate over $\mathscr{M}_{s}^{t}$ to obtain

$$
\begin{aligned}
& \text { LHS }=\int_{\mathscr{M}_{j}^{\prime}}\left|\partial_{t} u\right|^{2} \varphi^{2}+\alpha_{1} \int_{\mathscr{N}_{s}^{j}} \frac{d}{d t}\left(e(u) \varphi^{2}\right)-\alpha_{2} \int_{\mathscr{M}^{T}} \partial_{t} u(\phi(u) \times(\Delta u+h)) \varphi^{2} \\
& =\int_{\mathscr{M}_{s}^{t}}\left|\partial_{t} u\right|^{2} \varphi^{2}+\alpha_{1} \int_{\mathscr{M}_{s}^{\prime}} \frac{d}{d t}\left(e(u) \varphi^{2}\right)-\frac{\alpha_{2}^{2}}{\alpha_{1}^{2}+\alpha_{2}^{2}} \int_{\mathscr{M}_{s}^{t}}\left|\partial_{t} u\right|^{2} \varphi^{2} \\
& =\frac{\alpha_{1}^{2}}{\alpha_{1}^{2}+\alpha_{2}^{2}} \int_{\mathscr{N}_{s}^{\prime}}\left|\partial_{t} u\right|^{2} \varphi^{2}+\alpha_{1} \int_{\mathscr{M}_{s}^{\prime}} \frac{d}{d t}\left(e(u) \varphi^{2}\right) \text {, } \\
& \text { RHS } \leq \alpha_{1} \int_{\mathscr{K}_{s}^{\prime}}\left|\nabla u \| \partial_{t} u\right||\nabla \varphi| \varphi+\frac{\alpha_{1}^{2}}{2\left(\alpha_{1}^{2}+\alpha_{2}^{2}\right)} \int_{\mathscr{K}_{s}^{\prime}}\left|\partial_{t} u\right|^{2} \varphi^{2}+C \int_{\mathscr{M}_{j}^{\prime}}|h|^{2} \varphi^{2} \\
& \leq \frac{\alpha_{1}^{2}}{\alpha_{1}^{2}+\alpha_{2}^{2}} \int_{\mathscr{M}_{s}^{\prime}}\left|\partial_{t} u\right|^{2} \varphi^{2}+C R^{-2} \int_{\mathscr{M}_{s}^{\prime}}|\nabla u|^{2}+C \int_{\mathscr{M}_{s}^{!}}|h|^{2} \varphi^{2} .
\end{aligned}
$$


Hence

$$
\begin{aligned}
E_{R}(u(\cdot, t) ; x) & \leq \int_{\mathscr{K}} e(u(\cdot, t)) \varphi^{2}=\int_{\mathscr{N}} e\left(u_{0}\right) \varphi^{2}+\int_{\mathscr{K}_{s}^{t}} \frac{d}{d t}\left(e(u) \varphi^{2}\right) \\
& \leq \int_{\mathscr{K}} e\left(u_{0}\right) \varphi^{2}+C R^{-2} \int_{0}^{t} E(u(\cdot, t))+C \int_{\mathscr{A}_{0}^{\prime}}|h|^{2} \varphi^{2} \\
& \leq E_{2 R}\left(u_{0} ; x\right)+C R^{-2}\left(E_{0}+C H_{0}\right) t+C \int_{\mathscr{K}_{0}^{\prime}}|h|^{2} \varphi^{2} \\
& \leq E_{2 R}\left(u_{0} ; x\right)+C \frac{t}{R^{2}}\left(E_{0}+H_{*}\right),
\end{aligned}
$$

where $H_{*}=\left(H_{1}+\left\|h_{0}\right\|_{L^{2}}^{2}+H_{0}\right) R_{0}^{2}$. To obtain the last step in inequality (2.12), we use (2.5) and note that

$$
\begin{aligned}
& \int_{\mathscr{K}}|h(\cdot, t)|^{2}=\int_{\mathscr{K}}\left|\int_{0}^{t} \partial_{t} h(\cdot, t)+h(\cdot, 0)\right|^{2} \leq 2 \int_{\mathscr{K}}\left|\int_{0}^{t} \partial_{t} h(\cdot, t)\right|^{2}+2 \int_{\mathscr{K}^{\prime}}\left|h_{0}\right|^{2} \\
& \leq 2 \int_{\mathscr{K}} \int_{0}^{t} 1^{2} \int_{0}^{t}\left|\partial_{t} h(\cdot, t)\right|^{2}+2 \int_{\mathscr{K}}\left|h_{0}\right|^{2} \leq 2 H_{1} t+\left\|h_{0}\right\|_{L^{2}}^{2}, \\
& \int_{\mathscr{M}^{t}}|h|^{2} \varphi^{2} \leq \int_{\mathscr{K}^{t}}|h|^{2}=\int_{0}^{t} \int_{\mathscr{K}^{\prime}}|h(\cdot, t)|^{2} \leq \int_{0}^{t}\left(2 H_{1} t+\left\|h_{0}\right\|_{L^{2}}^{2}\right)=H_{1} t^{2}+\left\|h_{0}\right\|_{L^{2}}^{2} t,
\end{aligned}
$$

and hence $\int_{\mathscr{M}^{t}}|h|^{2} \varphi^{2} \leq \min \left\{H_{1} t^{2}+\left\|h_{0}\right\|_{L^{2}}^{2} t, H_{0}\right\}$.

For a solution $u \in V\left(\mathscr{M}^{T} ; \mathscr{N}\right)$ of $(1.1)$, and any $R \in\left(0, R_{0}\right]$, let $\varepsilon(R)=$ $\varepsilon(R ; u, T)=\sup _{(x, t) \in \mathscr{K}^{r}} E_{R}(u(\cdot, t) ; x)$, and in the sequel let $R$ be small enough to satisfy $\varepsilon(R) \leq \varepsilon_{0}$, where $\varepsilon_{0}=\varepsilon_{0}(\mathscr{M}, \mathscr{N})$ will be determined in Lemma 4-Lemma 7 .

LEMMA 4. There exists a constant $\varepsilon_{0}>0$ such that for any solution $u \in V\left(\mathscr{M}^{T} ; \mathscr{N}\right)$ of (1.1), and any $R \in\left(0, R_{0}\right]$, the estimate $\int_{\mathscr{M}^{T}}\left|\nabla^{2} u\right|^{2} \leq C\left(E_{0}+H_{0}\right)\left(1+T R^{-2}\right)$ holds provided that $\varepsilon(R) \leq \varepsilon_{0}$.

ProOF. Multiplying equation (1.3) by $\Delta u$ and integrating over $\mathscr{M}^{T}$, and by virtue of $(\phi(u) \times \Delta u) \Delta u=0$ and $|\phi(u) \times(\phi(u) \times h)+\phi(u) \times h| \leq C|h|$, we get

$$
\begin{aligned}
& \frac{1}{2} \int_{\mathscr{M}^{\tau}} \frac{d}{d t}(\nabla u, \nabla u)+\alpha_{1} \int_{\mathcal{M}^{T}}|\Delta u|^{2} \\
& \leq C \int_{\mathscr{K}^{T}}|\Delta u \| \nabla u|^{2}+C \int_{\mathscr{K}^{T}}|h||\Delta u| \\
& \leq \frac{\alpha_{1}}{4} \int_{\mathscr{M}^{T}}|\Delta u|^{2}+C \int_{\mathscr{N}^{T}}|\nabla u|^{4}+\frac{\alpha_{1}}{4} \int_{\mathscr{N}^{T}}|\Delta u|^{2}+C \int_{\mathscr{N}^{T}}|h|^{2} \\
& \leq \frac{\alpha_{1}}{2} \int_{\mathscr{N}^{T}}|\Delta u|^{2}+C \int_{\mathscr{N}^{T}}|\nabla u|^{4}+C \int_{\mathscr{M}^{T}}|h|^{2} \text {. }
\end{aligned}
$$


By Remark 4 and the definition of $\varepsilon(R)$ we get the following inequality:

$$
\int_{M^{T}}|\nabla u|^{4} \leq C \varepsilon(R)\left(\int_{\mathscr{M}^{T}}\left|\nabla^{2} u\right|^{2}+C \frac{T}{R^{2}}\left(E_{0}+H_{0}\right)\right) .
$$

From (2.13)-(2.14) if we let $\varepsilon(R) \leq \varepsilon_{0}$ be sufficiently small we get

$$
\begin{aligned}
\frac{1}{2} \int_{\mathscr{M}^{T}} & \frac{d}{d t}(\nabla u, \nabla u)+\frac{\alpha_{1}}{2} \int_{\mathscr{N}^{T}}|\Delta u|^{2} \\
\leq & C H_{0}+\frac{\alpha_{1}}{4}\left(\int_{\mathscr{M}^{T}}\left|\nabla^{2} u\right|^{2}+C \frac{T}{R^{2}}\left(E_{0}+H_{0}\right)\right) \\
\leq & \frac{\alpha_{1}}{4}\left(\int_{\mathscr{N}^{T}}\left|\nabla^{2} u\right|^{2}+C\left(1+\frac{T}{R^{2}}\right)\left(E_{0}+H_{0}\right)\right) .
\end{aligned}
$$

Moreover, integrating by parts yields the estimate

$$
\int_{\mathscr{M}^{T}}|\Delta u|^{2} \geq \int_{\mathscr{M}^{T}}\left|\nabla^{2} u\right|^{2}-C \int_{\mathscr{M}^{T}}|\nabla u|^{2},
$$

where the coefficient $C$ on the right-hand side results from the differentials of the metric $\gamma$. Therefore from (2.15)-(2.16) and (2.6) we obtain

$$
\int_{\mathscr{M}^{T}}\left|\nabla^{2} u\right|^{2} \leq C\left(E_{0}+H_{0}\right)\left(1+T R^{-2}\right)
$$

In order to state the pointwise a-priori estimates of the solution we now derive the uniform local estimates of $\int_{\mathscr{M}_{t}^{t}}|\nabla u|^{4}$ with respect to time $t$. The proof of the following lemma is similar to the one of $[15$, Lemma 3.8$]$ and will be omitted.

LEMMA 5. For any positive numbers $\epsilon$ and $\tau$, and any $R \in\left(0, R_{0}\right]$, there exists a number $\delta>0$ such that for any solution $u \in V\left(\mathscr{M}^{T} ; \mathscr{N}\right)$ of $(1.1)$ and any $I \subset[\tau, T]$ with measure $|I|<\delta$, the estimate $\int_{I}\left(\int_{\mathscr{M}}|\nabla u|^{4}\right)<\epsilon$ holds provided that $\varepsilon(R) \leq \varepsilon_{0}$ and $u_{0}$, $h$ satisfy (1.2).

In the next lemma, we prove the closure property of the solutions of the LandauLifshitz equations in $V\left(\mathscr{M}^{T} ; \mathscr{N}\right)$.

LEMMA 6. Let $u_{m} \in V\left(\mathscr{M}^{T} ; \mathscr{N}\right)$ be solutions of $(1.1)$ issuing from a set of initial data $u_{m, 0}$ with $u_{m, 0} \rightarrow u_{0}$, and suppose there exists some $R \in\left(0, R_{0}\right]$ such that $\varepsilon\left(R ; u_{m}, T\right) \leq \varepsilon_{0}$ for every $m \in N$. Then $\left\{u_{m}\right\}$ has a subsequence which converges to $u$ in $V\left(\mathscr{M}^{T} ; \mathscr{N}\right)$ such that $u$ solves $(1.1)$ with initial data $u_{0}$.

PRoof. We may suppose $u_{m} \rightarrow u$ a.e., $\partial_{t} u_{m} \rightarrow \partial_{t} u, \nabla^{2} u_{m} \rightarrow \nabla^{2} u$ weakly in $L^{2}\left(\mathscr{M}^{T}\right)$ and $\nabla u_{m} \rightarrow \nabla u$ strongly in $L^{2}\left(\mathscr{M}^{T}\right)$. Let

$$
v_{m}=u_{m}-u \quad \text { and } \quad\left|\nabla U_{m}\right|=\left|\nabla u_{m}\right|+|\nabla u| .
$$


From (1.1) we have

$$
\begin{aligned}
\partial_{t} v_{m}- & \alpha_{1} \Delta v_{m}-\alpha_{2}\left(\phi\left(u_{m}\right) \times \Delta v_{m}\right) \\
= & \alpha_{1}\left(A\left(u_{m}\right)\left(\nabla u_{m}, \nabla u_{m}\right)-A(u)\left(\nabla u_{m}, \nabla u_{m}\right)+A(u)\left(\nabla v_{m}, \nabla u_{m}\right)\right. \\
& \left.+A\left(u_{m}\right)\left(\nabla u, \nabla v_{m}\right)\right)+\alpha_{2}\left(\phi\left(u_{m}\right)-\phi(u)\right) \times \Delta u \\
& +\alpha_{1} \phi\left(u_{m}\right) \times\left(\phi\left(u_{m}\right) \times h\right)-\alpha_{1} \phi(v) \times(\phi(v) \times h) \\
& +\alpha_{2} \phi\left(u_{m}\right) \times h-\alpha_{2} \phi(v) \times h=\mathrm{I}+\mathrm{II}+\mathrm{III},
\end{aligned}
$$

where

$$
\begin{aligned}
|\mathrm{I}|= & \mid \alpha_{1}\left(A\left(u_{m}\right)\left(\nabla u_{m}, \nabla u_{m}\right)-A(u)\left(\nabla u_{m}, \nabla u_{m}\right)+A(u)\left(\nabla v_{m}, \nabla u_{m}\right)\right. \\
& \left.+A\left(u_{m}\right)\left(\nabla u, \nabla v_{m}\right)\right) \mid \leq C\left(\left|\nabla U_{m}\right|^{2}\left|v_{m}\right|+\left|\nabla v_{m}\right|\left|\nabla U_{m}\right|\right) \\
|\mathrm{II}|= & \left|\alpha_{2}\left(\phi\left(u_{m}\right)-\phi(u)\right) \times \Delta u\right| \leq C|\Delta u|\left|v_{m}\right| \\
|\mathrm{III}|= & \mid\left(\alpha_{1} \phi\left(u_{m}\right) \times\left(\phi\left(u_{m}\right) \times h\right)-\alpha_{1} \phi(v) \times(\phi(v) \times h)\right) \\
& +\left(\alpha_{2} \phi\left(u_{m}\right) \times h-\alpha_{2} \phi(v) \times h\right)|\leq C| h|| v_{m} \mid .
\end{aligned}
$$

Multiplying I, II and III by $\Delta v_{m}$ and integrating, by Young's inequality, we get

$$
\begin{aligned}
\int_{\mathscr{M}^{T}}\left|I \cdot \Delta v_{m}\right| & \leq C \int_{\mathscr{N}^{T}}\left(\left|\nabla U_{m}\right|^{2}\left|v_{m}\right|\left|\Delta v_{m}\right|+\left|\nabla v_{m}\right|\left|\nabla U_{m}\right|\left|\Delta v_{m}\right|\right) \\
& \leq \frac{1}{4} \int_{\mathscr{M}^{T}}\left|\Delta v_{m}\right|^{2}+C \int_{\mathscr{N}^{T}}\left(\left|\nabla U_{m}\right|^{4}\left|v_{m}\right|^{2}+\left|\nabla v_{m}\right|^{2}\left|\nabla U_{m}\right|^{2}\right) ; \\
\int_{\mathscr{M}^{T}}\left|\mathrm{II} \cdot \Delta v_{m}\right| & \leq C \int_{\mathscr{N}^{T}}|\Delta u|\left|\Delta v_{m}\right|\left|v_{m}\right| \leq \frac{1}{4} \int_{\mathscr{N}^{T}}\left|\Delta v_{m}\right|^{2}+C \int_{\mathscr{M}^{T}}\left|v_{m}\right|^{2}|\Delta u|^{2} ; \\
\int_{\mathscr{M}^{T}}\left|\mathrm{III} \cdot \Delta v_{m}\right| & \leq C \int_{\mathscr{M}^{T}}|h|\left|v_{m}\right|\left|\Delta v_{m}\right| \leq \frac{1}{4} \int_{\mathscr{N}^{T}}\left|\Delta v_{m}\right|^{2}+C \int_{\mathscr{M}^{T}}\left|v_{m}\right|^{2}|h|^{2} .
\end{aligned}
$$

Hence it follows from (2.17) that

$$
\begin{aligned}
\frac{1}{2}\left(\int_{\mathscr{K}}\left|\nabla v_{m}(\cdot, T)\right|^{2}-\int_{\mathscr{K}}\left|\nabla v_{m}(\cdot, 0)\right|^{2}\right)+\int_{\mathscr{K}^{T}}\left|\Delta v_{m}\right|^{2} \\
\leq \leq \frac{3}{4} \int_{\mathscr{N}^{T}}\left|\Delta v_{m}\right|^{2}+\int_{\mathscr{M}^{T}}\left|v_{m}\right|^{2}|\Delta u|^{2} \\
\quad+C \int_{\mathscr{N}^{T}}\left(\left|\nabla U_{m}\right|^{4}\left|v_{m}\right|^{2}+\left.\nabla v_{m}\right|^{2}\left|\nabla U_{m}\right|^{2}\right)+C \int_{\mathscr{M}^{T}}\left|v_{m}\right|^{2}|h|^{2}
\end{aligned}
$$

Rearranging (2.18), and by (2.16),

$$
\begin{aligned}
\sup _{0 \leq t \leq T} & \int_{\mathscr{M}}\left|\nabla v_{m}(\cdot, t)\right|^{2}+\int_{\mathscr{M}^{T}}\left|\nabla^{2} v_{m}\right|^{2} \\
& \leq C \int_{\mathscr{N}^{T}}\left|\nabla v_{m}(\cdot, 0)\right|^{2}+C \int_{\mathscr{M}^{T}}\left(\left|\nabla U_{m}\right|^{4}\left|v_{m}\right|^{2}+\left|\nabla v_{m}\right|^{2}\left|\nabla U_{m}\right|^{2}\right)
\end{aligned}
$$




$$
+C \int_{\mathcal{K}^{T}}\left|v_{m}\right|^{2}|h|^{2} \rightarrow 0 \text { as } m \rightarrow \infty \text {. }
$$

Similarly, multiplying the two sides of (2.17) by $\partial_{t} v_{m}$ and integrating over $\mathscr{M}^{T}$ gives, as $m \rightarrow \infty$,

$$
\begin{aligned}
\int_{\mathscr{K}}\left|\partial_{t} v_{m}\right|^{2} \leq & C_{1} \int_{\mathscr{N}^{T}}\left|\nabla v_{m}(\cdot, 0)\right|^{2} \\
& +C_{2} \int_{\mathscr{K}^{T}}\left(\left|\nabla U_{m}\right|^{4}\left|v_{m}\right|^{2}+\left.\left|\nabla v_{m}\right|^{2}\left|\nabla U_{m}+\right| v_{m}\right|^{2}|h|^{2}\right) \rightarrow 0 .
\end{aligned}
$$

Hence, $u_{m} \rightarrow u$ strongly in $V\left(\mathscr{M}^{T} ; \mathscr{N}\right)$.

Next we will use Hölder estimate to show the uniform continuity of the regular solutions.

LEMMA 7. Let $u \in V\left(\mathscr{M}^{T} ; \mathscr{N}\right) \bigcap_{\tau>0} C^{2}\left(\mathscr{M}_{\tau}^{T} ; \mathscr{N}\right)$ be a regular solution of $(1.1)$. Then for any $\tau>0$ the Hölder norms of $u$ and its derivatives may be estimated uniformly on $\mathscr{M}_{\tau}^{T}$ by quantities involving $E_{0}, H_{0}, H_{\infty}, H_{1}, \tau, T$ and $R$, provided $\varepsilon(R) \leq \varepsilon_{0}$.

PROOF. Multiplying (1.3) by $\Delta u$ and integrating over $\mathscr{M}$, by virtue of $\Delta u(\phi(u) \times$ $\Delta u)=0$, we get

$$
\begin{aligned}
\alpha_{1} \int_{\mathscr{M}}|\Delta u|^{2}= & \int_{\mathscr{H}} \Delta u \partial_{t} u-\alpha_{1} \int_{\mathscr{M}} A(u)(\nabla u, \nabla u) \Delta u \\
& +\int_{\mathscr{M}}\left(\alpha_{1} \phi(u) \times(\phi(u) \times h)-\alpha_{2} \phi(u) \times h\right) \Delta u .
\end{aligned}
$$

By Young's inequality

$$
\begin{aligned}
& \int_{\mathscr{N}}(\Delta u) \partial_{t} u \leq \frac{\alpha_{1}}{4} \int_{\mathscr{A}}|\Delta u|^{2}+C \int_{\mathscr{N}}\left|\partial_{t} u\right|^{2} \\
& \int_{\mathscr{N}} A(u)(\nabla u, \nabla u) \Delta u \leq C \int_{\mathscr{M}}|\nabla u|^{2}|\Delta u| \leq \frac{1}{4} \int_{\mathscr{M}}|\Delta u|^{2}+C \int_{\mathscr{K}}|\nabla u|^{4} \\
& \int_{\mathscr{K}}\left(\alpha_{1} \phi(u) \times(\phi(u) \times h)+\alpha_{2} \phi(u) \times h\right) \Delta u \\
& \leq C \int_{\mathscr{K}}|\Delta u||h| \leq \frac{\alpha_{1}}{4} \int_{\mathscr{K}}|\Delta u|^{2}+C \int_{\mathscr{K}}|h|^{2}
\end{aligned}
$$

From (2.19)-(2.22) we get

$$
\int_{\mathscr{\mu}}|\Delta u|^{2} \leq C \int_{\mathscr{\mu}}\left(|\nabla u|^{4}+\left|\partial_{t} u\right|^{2}+|h|^{2}\right) .
$$


Next, differentiate the equation (1.3) with respect to $t$, multiply $\partial_{t} u$ on both sides and then integrate over $\mathscr{M}_{s}^{\prime}, \tau \leq s<t \leq T$, we get

$$
\begin{aligned}
& \int_{\mathcal{M}_{!}^{\prime}} \partial_{i} u \partial_{t t}^{2} u=\alpha_{1} \int_{\mathscr{M}_{s}^{\prime}} \partial_{t} u \Delta \partial_{t} u+\alpha_{1} \int_{\mathscr{N}_{!}^{\prime}} \partial_{t} u \partial_{t}(A(u)(\nabla u, \nabla u)) \\
& -\alpha_{1} \int_{\mathscr{N}_{s}^{\prime}} \partial_{t} u \partial_{t}(\phi(u) \times(\phi(u) \times h) \\
& \left.+\alpha_{2} \int_{\mathscr{M}_{s}} \partial_{t} u \partial_{t}(\phi(u) \times h)\right)+\alpha_{2} \int_{\mathcal{M}_{t}^{t}} \partial_{t} u \partial_{t}(\phi(u) \times \Delta u) .
\end{aligned}
$$

For the right-hand side of (2.24), first we note that

$$
\begin{aligned}
\left|\partial_{t}(A(u)(\nabla u, \nabla u))^{l}\right| & =\left|\partial_{t}\left(\gamma^{a b} \Gamma_{i j}^{l}(u) \frac{\partial}{\partial x^{a}} u^{i} \frac{\partial}{\partial x^{b}} u^{j}\right)\right| \\
& \leq C\left|\nabla \partial_{t} u\|\nabla u|+C| \nabla u\| \nabla u \| \partial_{t} u\right|,
\end{aligned}
$$

and

$$
\begin{aligned}
& \mid-\alpha_{1} \int_{\mathscr{M}_{s}^{\prime}} \partial_{t} u \partial_{t}\left(\phi(u) \times(\phi(u) \times h)+\alpha_{2} \int_{\mathscr{N}_{s}^{\prime}} \partial_{t} u\left(\partial_{t} \phi(u) \times h\right) \mid\right. \\
& \quad \leq C \int_{\mathscr{N}_{s}^{\prime}}\left(\left|\partial_{t} u\left\|\partial_{t} \phi(u)\right\| h\right|+\left|\partial_{t} u \| \partial_{t} h\right|\right) \\
& \quad \leq C\left(1+h_{\infty}\right) \int_{\mathscr{K}_{s}^{\prime}}\left(\left|\partial_{t} u\right|^{2}+\left|\partial_{t} u\right|\right)\left|\partial_{t} h\right| .
\end{aligned}
$$

Also,

(2.27)

$$
\begin{aligned}
&\left|\int_{\mathscr{M}_{s}^{\prime}} \alpha_{2} \partial_{t} u \partial_{t}(\phi(u) \times \Delta u)\right| \\
&=\left|\int_{\mathscr{M}_{s}^{\prime}} \alpha_{2} \partial_{t} u \partial_{t}(\nabla(\phi(u) \times \nabla u)-\nabla \phi(u) \times \nabla u)\right| \\
&=\mid \int_{\mathscr{N}_{s}^{\prime}} \alpha_{2} \partial_{t} u\left(\nabla\left(\partial_{t} \phi(u) \times \nabla u\right)+\nabla\left(\phi(u) \times \nabla \partial_{t} u\right)\right) \\
&-\int_{\mathscr{M}_{s}^{\prime}} \alpha_{2} \partial_{t} u\left(\nabla \partial_{t} \phi(u) \times \nabla u+\nabla \phi(u) \times \nabla \partial_{t} u\right) \mid \\
& \leq C \int_{\mathscr{M}_{t}^{\prime}}\left(\left|\partial_{t} u\left\|\nabla \partial_{t} u\right\| \nabla u\right|+\left|\partial_{t} u\right|^{2}|\nabla u|^{2}\right) \\
& \leq \frac{\alpha_{1}}{4} \int_{\mathscr{M}_{s}^{\prime}}\left|\partial_{t} \nabla u\right|^{2}+C \int_{\mathscr{K}_{s}^{\prime}}\left|\partial_{t} u\right|^{2}|\nabla u|^{2} .
\end{aligned}
$$

From (2.24)-(2.27) we obtain

$$
\frac{1}{2} \int_{\mathscr{K}_{s}^{\prime}} \partial_{t}\left|\partial_{t} u\right|^{2}+\alpha_{1} \int_{\mathscr{M}_{s}^{\prime}}\left|\nabla \partial_{t} u\right|^{2}
$$




$$
\begin{aligned}
\leq & C \int_{\mathscr{N}_{s}^{\prime}}\left(\left|\partial_{t} u\right|^{2}|\nabla u|^{2}+\left|\partial_{t} u\right||\nabla u|\left|\nabla \partial_{t} u\right|\right) \\
& +\alpha_{2} \int_{\mathscr{K}_{s}^{\prime}}\left|\left(\phi(u) \times \Delta \partial_{t} u\right) \partial_{t} u\right|+C \int_{\mathscr{K}_{s}^{\prime}}\left(\left|\partial_{t} u\right|^{2}+\left|\partial_{t} h\right|^{2}+|h|^{2}\right) \\
\leq & \frac{\alpha_{1}}{2} \int_{\mathscr{K}_{s}^{\prime}}\left|\partial_{t} \nabla u\right|^{2}+C \int_{\mathscr{K}_{s}^{\prime}}\left|\partial_{t} u\right|^{2}|\nabla u|^{2}+C \int_{\mathscr{K}_{s}^{\prime}}\left(\left|\partial_{t} u\right|^{2}+\left|\partial_{t} h\right|^{2}+|h|^{2}\right) .
\end{aligned}
$$

For $|t-s| \leq \delta$ sufficiently small, by the proof of [15, Lemma 3.10], we have

$$
\begin{aligned}
\int_{\mathscr{N}}\left|\partial_{t} u(\cdot, t)\right|^{2} & \leq C_{1} \inf _{0 \leq t-\delta \leq s \leq t} \int_{\mathscr{M}}\left|\partial_{t} u(\cdot, s)\right|^{2}+C_{2} \int_{\mathscr{M}_{s}}\left(\left|\partial_{t} u\right|^{2}+\left|\partial_{t} h\right|^{2}+|h|^{2}\right) \\
& =C_{2} \inf _{0 \leq t-\delta \leq s \leq t} \int_{\mathscr{K}}\left|\partial_{t} u(\cdot, s)\right|^{2}+C_{3},
\end{aligned}
$$

where the constants $C_{1}, C_{2}, C_{3}$ depend on $E_{0}, H_{0}, H_{1}, H_{\infty}, \tau, T, R, \delta$. It follows that

$$
\left.\operatorname{ess} \sup _{\tau \leq t \leq T}\left|\int_{\mathscr{A}}\right| \partial_{t} u\right|^{2} \leq C \int_{\mathcal{N}_{s}^{t}}\left|\partial_{t} u\right|^{2} \leq C\left(E_{0}+H_{0}+H_{1}\right)\left(1+\tau^{-1}\right) .
$$

So from $[15,(3.6)],(2.16),(2.23)$, and (2.28), we get

$$
\int_{\|}\left|\nabla^{2} u\right|^{2} \leq C\left(E_{0}+H_{0}+H_{1}\right)\left(1+\tau^{-1}+R^{-2}\right)
$$

provided that $\varepsilon(R) \leq \varepsilon_{0}$. The remainder of the proof which leads to the estimates of the Hölder norms of $u$ and its derivatives is similar to that of [6, Lemma 3.8].

We now prove the uniqueness of the solution in the class $V\left(\mathscr{M}_{\tau}^{T} ; \mathscr{N}\right)$.

LEMMA 8. Suppose $u_{1}, u_{2} \in V\left(\mathscr{M}^{T} ; \mathscr{N}\right)$ are solutions to the Landau-Lifshitz equation (1.1) with the same initial value $u_{1}(\cdot, 0)=u_{2}(\cdot, 0)=u_{0}$. Then $u_{1}=u_{2}$ in $\mathscr{M}^{T}$.

PROOF. Let $v=u_{1}-u_{2},|\nabla U|=\left|\nabla u_{1}\right|+\left|\nabla u_{2}\right|$. From (1.1) we get

$$
\begin{aligned}
\mid \partial_{t} v- & \alpha_{1} \Delta v+\alpha_{2} \phi\left(u_{1}\right) \times \Delta u_{1}-\alpha_{2} \phi\left(u_{2}\right) \times \Delta u_{2} \mid \\
\leq & \alpha_{1}\left|A\left(u_{1}\right)\left(\nabla u_{1}, \nabla u_{1}\right)-A\left(u_{2}\right)\left(\nabla u_{2}, \nabla u_{2}\right)\right|+C|v||h| \\
= & \alpha_{1}\left(\left|A\left(u_{1}\right)\left(\nabla u_{1}, \nabla u_{1}\right)-A\left(u_{2}\right)\left(\nabla u_{1}, \nabla u_{1}\right)\right|+C|v||h|\right. \\
& \left.+\left|A\left(u_{2}\right)\left(\nabla u_{1}, \nabla u_{1}-\nabla u_{2}\right)\right|+\left|A\left(u_{2}\right)\left(\nabla u_{1}-\nabla u_{2}, \nabla u_{2}\right)\right|\right) \\
\leq & C\left(|v||\nabla U|^{2}+|\nabla U||\nabla v|\right)+C|v||h| .
\end{aligned}
$$

If we multiply (2.29) by $v$, and integrate on $\mathscr{M}^{t}$, then

$$
\frac{1}{2} \int_{\mathscr{K}}(v(\cdot, t))^{2}+\alpha_{1} \int_{\mathcal{K}^{t}}(\nabla v, \nabla v)
$$




$$
\begin{aligned}
\leq & C_{1} \int_{\mathscr{M}^{\prime}}\left(|v|^{2}|\nabla U|^{2}+|v||\nabla U||\nabla v|\right)+\int_{\mathscr{N}^{\prime}} C|v||h| \\
& +C_{2} \int_{\mathscr{N}^{\prime}}\left|\left(\phi\left(u_{1}\right) \times \Delta u_{1}-\phi\left(u_{2}\right) \times \Delta u_{2}\right) v\right| .
\end{aligned}
$$

We note that

$$
\begin{aligned}
& \int_{\mathscr{M}}\left|\left(\phi\left(u_{1}\right) \times \Delta u_{1}-\phi\left(u_{2}\right) \times \Delta u_{2}\right) v\right| \\
&=\int_{\mathscr{M}}\left|\left(\left(\phi\left(u_{1}\right)-\phi\left(u_{2}\right)\right) \times \Delta u_{1}+\phi\left(u_{2}\right) \times \Delta v\right) v\right| \\
& \leq \int_{\mathscr{K}}\left|\left(\left(\phi\left(u_{1}\right)-\phi\left(u_{2}\right)\right) \times \Delta u_{1}\right) v\right|+\left|\left(\phi\left(u_{2}\right) \times \Delta v\right) v\right| \\
&= \int_{\mathscr{M}}\left|\left(\left(\phi\left(u_{1}\right)-\phi\left(u_{2}\right)\right) \times v\right) \Delta u_{1}\right|+\left|\left(\phi\left(u_{2}\right) \times v\right) \Delta v\right| \\
&= \int_{\mathscr{M}}\left|\left(\nabla\left(\left(\phi\left(u_{1}\right)-\phi\left(u_{2}\right)\right) \times v\right)\right) \nabla u_{1}\right|+\left|\nabla\left(\phi\left(u_{2}\right) \times v\right) \nabla v\right| \\
& \leq\left.\int_{\mathscr{M}}\left|\left(\nabla\left(\phi\left(u_{1}\right)-\phi\left(u_{2}\right)\right) \times v\right) \nabla u_{1}\right|+\mid\left(\phi\left(u_{1}\right)-\phi\left(u_{2}\right)\right) \times \nabla v\right) \nabla u_{1} \mid \\
&+\left|\left(\nabla \phi\left(u_{2}\right) \times v\right) \nabla v\right| \\
& \leq C \int_{\mathscr{M}^{\prime}}|v|^{2}|\nabla U|^{2}+\int_{\mathscr{M}^{\prime}}|\nabla U||v||\nabla v| .
\end{aligned}
$$

Combining (2.30)-(2.31), we get

$$
\begin{aligned}
\frac{1}{2} \int_{\mathscr{N}^{\prime}} & (v(\cdot, t))^{2}+\alpha_{1} \int_{\mathscr{N}^{t}}(\nabla v, \nabla v) \\
\leq & C \int_{\mathscr{M}^{t}}\left(|v|^{2}|\nabla U|^{2}+|v||\nabla U||\nabla v|\right)+C \int_{\mathscr{N}^{\prime}}|v(\cdot, t)|^{2}|h| \\
& \leq C \int_{\mathscr{M}^{\prime}}|v|^{2}|\nabla U|^{2}+\frac{\alpha_{1}}{2} \int_{\mathscr{N}^{\prime}}|\nabla v|^{2}+C \int_{\mathscr{N}^{\prime}}|h||v|^{2} .
\end{aligned}
$$

By Lemma 2, for any $S>0$, there exists $t_{0} \in[0, S]$ such that

$$
\sup _{0 \leq s \leq s} \int_{\mathscr{A}}\left|\partial_{t} u(\cdot, s)\right|^{2}=\int_{\mathscr{A}}\left|\partial_{t} u\left(\cdot, t_{0}\right)\right|^{2}
$$

If we choose $S$ less than $1 /\left(4 C H_{\infty}\right)$, then

$$
C \int_{\mathscr{M}^{s}}|h||v|^{2} \leq \frac{1}{4} \int_{\mathscr{M}}\left|v\left(\cdot, t_{0}\right)\right|^{2} .
$$

Finally, following the proof of [15, Lemma 3.12], we obtain

$$
\frac{1}{4} \int_{\mathscr{M}}\left(v\left(\cdot, t_{0}\right)\right)^{2}+\frac{\alpha_{1}}{2} \int_{\mathscr{N}^{s}}|\nabla v|^{2} \leq 0 .
$$


Thus, it follows that $v=0$ in $\mathscr{M}^{s}$. A continuation argument shows that $u_{1}=u_{2}$ in $\mathscr{M}^{T}$.

As for the local existence of the solution of (1.1), we use an idea of Hamilton's [7] and derive the local existence result when the initial data $u_{0}$ is smooth.

LEMMA 9. For the problem (1.1), let $u_{0}$ be smooth. Then, there exists a unique (local) solution $u \in W^{2,2}(\mathscr{M} \times[0, \varepsilon], \mathscr{N})$ for some $\varepsilon>0$.

PROOF. First we define a nonlinear map $L: W^{2,2}\left(\mathscr{M}^{\omega}\right) \rightarrow L^{2}\left(\mathscr{M}^{\omega}\right)$ as follows:

$$
\begin{aligned}
L u= & \partial_{t} u+\alpha_{1} \phi(u) \times(\phi(u) \times(\Delta u+h))-\alpha_{2} \phi(u) \times(\Delta u+h) \\
= & \partial_{t} u-\alpha_{1} \Delta u-\alpha_{1} A(u)(\nabla u, \nabla u) \\
& +\alpha_{1} \phi(u) \times(\phi(u) \times h)-\alpha_{2} \phi(u) \times(\Delta u+h) .
\end{aligned}
$$

For a smooth map $u$, the derivative of $L$ at $u$ is given by

$$
D L(u) f=\partial_{t} f-\alpha_{1} \Delta f-\alpha_{2} \phi(u) \times \Delta f-a(u, h) \nabla f-b(u, h) f,
$$

where $a(u, h)$ and $b(u, h)$ are smooth matrix-valued functions.

Write $H f=\partial_{t} f-\alpha_{1} \Delta f-\alpha_{2} \phi\left(u_{0}\right) \times \Delta f$ and $K f=-a\left(u_{0}, h\right) \nabla f-b\left(u_{0}, h\right) f$. Observe that for any $\xi \neq 0$, we have $\alpha_{1} \xi \cdot \xi+\alpha_{2}\left(\phi\left(u_{0}\right) \times \xi\right) \cdot \xi=\alpha_{1} \xi \cdot \xi>0$ and hence $H$ is a uniformly parabolic quasilinear operator. Therefore the map $f \mapsto H f$ defines an isomorphism from $W^{2,2}(\mathscr{M} \times[0, \omega])$ onto $L^{2}(\mathscr{M} \times[0, \omega])$. Also, since $K: W^{2,2}(\mathscr{M} \times[0, \omega]) \rightarrow L^{2}(\mathscr{M} \times[0, \omega])$ is compact, by Fredholm theory, the indices of the maps $H$ and $H+K$ are both equal to 0 , that is, the dimensions of the kernel and cokernel of $H+K$ are finite and equal. On the other hand, by an argument similar to that of [6, Lemma 3.10], we know that the kernel of $H+K$ has dimension zero. Therefore, $D L\left(u_{0}\right)$ is an isomorphism. By the inverse function theorem the set of all $H\left(u_{0}+u^{*}\right)$ for $u^{*}$ in a neighbourhood of 0 covers a neighbourhood of $L\left(u_{0}\right)$ in $L^{2}(\mathscr{M} \times[0, \omega])$. If we choose $\varepsilon>0$ small enough, the function equal to 0 for $0 \leq t \leq \varepsilon$ and equal to $L\left(u_{0}\right)$ for $\varepsilon \leq t \leq \omega$ will be in this neighbourhood. So $\left.u\right|_{\mathscr{N}^{*}}$ solves the Landau-Lifshitz equation. Using the standard bootstrap method we can get the smoothness of the solution.

PROOF OF THEOREM 1. To complete the proof of Theorem 1, we proceed to deduce the existence of global almost smooth solutions. Given any initial data $u_{0} \in W^{1,2}(\mathscr{M})$, approximate $u_{0}$ by smooth maps $\left\{u_{0}^{m}\right\}_{m=1}^{\infty}$. By Lemma 9 there exists a sequence of local smooth solutions $\left\{u^{m}\right\}_{m=1}^{\infty}$ for (1.1) for which the estimates of Lemma 2-Lemma 7 are valid. Since $u_{0}^{m} \rightarrow u_{0}$ in $W^{1,2}(\mathscr{M})$, we can choose $R$ such that, for any $x \in \mathscr{M}$,

$$
E_{2 R}\left(u_{0}^{m} ; x\right) \leq \varepsilon_{0} / 2 .
$$


By Lemma 3, there exists $T_{1}$ of order at least $\varepsilon_{0} R_{0}^{2}$ and such that $\varepsilon\left(R ; u_{m}, T_{1}\right) \leq \varepsilon_{0}$ uniformly. Hence, by Lemma $7, u^{m}$ converges uniformly in $V\left(\mathscr{M}^{T_{1}} ; \mathscr{N}\right)$; moreover, by Lemma 6 , the limit is a solution of (1.1).

To verify the characterization condition (1.4) for the singular points, we proceed by contradiction. Let $T_{1}$ be the maximal existence time for a smooth solution starting from $u_{0}$ and suppose (1.4) is false, that is, for all $x \in \mathscr{M}$,

$$
\underset{t \rightarrow T_{1}^{-}}{\limsup } E_{R}(u(\cdot, t) ; x) \leq \varepsilon_{0} \quad \text { for all } R \in\left(0, R_{0}\right]
$$

Then, by the regularity estimates of Lemma $7, u$ may be continuously extended to the closed interval $\left[0, T_{1}\right]$ and $u\left(\cdot, T_{1}\right)$ is smooth. Hence by Lemma 9 the local smooth solution can be extended to a larger interval.

The finiteness of bubbles at fixed singular time $T_{1}$ follows from Lemma 3, (2.6) and the additivity of the energy. Indeed, (2.33) can fail at at most finitely many points. Suppose singularities occur at $\left\{x_{l}, T_{1}\right\}_{l=1}^{L_{1}}$. Then for any $R \in\left(0, R_{0}\right]$ and any compact submanifold $\mathscr{M}^{\prime} \subset \mathscr{M} \backslash \bigcup_{l=1}^{L_{1}} B_{R}\left(x_{l}\right)$, it follows from (2.9) that

$$
\begin{aligned}
E_{\mathscr{M}^{\prime}}\left(u\left(\cdot, T_{1}\right)\right) & \leq \liminf _{t \rightarrow T_{1}^{-}} E_{\mathscr{M}^{\prime}}(u(\cdot, t)) \\
& \leq \liminf _{t \rightarrow T_{1}^{-}} E_{\mathscr{K}}(u(\cdot, t))-\sum_{l=1}^{L_{1}} E_{R}\left(u(\cdot, t) ; x_{l}\right) \\
& \leq E_{\mathscr{M}}\left(u_{0}\right)+C \int_{0}^{T_{1}} \int_{\mathscr{M}}|h|^{2}-L \varepsilon_{0} .
\end{aligned}
$$

Letting $R \rightarrow 0$, and $\mathscr{M}^{\prime} \rightarrow \mathscr{M}$, we get

$$
E_{\mathscr{M}}\left(u\left(\cdot, T_{1}\right)\right) \leq E_{0}+C \int_{0}^{T_{1}} \int_{\mathscr{M}}|h|^{2}-L_{1} \varepsilon_{0} .
$$

To see global existence, we first note that $u(\cdot, t)$ is uniformly bounded in $W^{1,2}(\mathscr{M})$ (with respect to $t$ ). Thus, we can find a sequence $t_{i}$ such that $u\left(\cdot, t_{i}\right) \rightarrow u\left(\cdot, T_{1}\right)$ weakly in $W^{1,2}(\mathscr{M})$ and, by the energy estimate of Lemma $2, \nabla u(\cdot, t) \rightarrow \nabla u\left(\cdot, T_{1}\right)$ strongly in $L^{2}(\mathscr{M})$. Hence, $u$ may be continued to $u\left(\cdot, T_{1}\right)$ weakly in $W^{1,2}(\mathscr{M})$ with

$$
\int_{\mathscr{M}}\left|\nabla u\left(\cdot, T_{1}\right)\right|^{2} \leq \liminf _{t \rightarrow T_{1}^{-}} \int_{\mathscr{M}}|\nabla u(\cdot, t)|^{2} .
$$

Restart from $T_{1}$ with $u\left(\cdot, T_{1}\right)$ as the initial data. Iterating this for $T=T_{1}, T_{2}, \ldots$, we obtain the global solution.

Finally, repeating (2.34) for $i=1,2, \ldots$, we conclude that the number of singularities satisfies $\sum_{i} L_{i} \leq\left(E_{0}+C H_{0}\right) / \varepsilon_{0}<\infty$. 


\section{Singularity and energy identity}

In the previous section we showed the existence and uniqueness of almost smooth solutions of the Landau-Lifshitz equations. Such solutions may have finitely many singular points for given initial data in $W^{1,2}(\mathscr{M})$. Near these singular points, we can use the energy identity to describe the behaviour of the solutions. For the evolution of harmonic maps many results related to this issue have been obtained. Qing [12] established an energy identity and showed the pointwise bubble tree convergence for the evolution of harmonic maps in the case where the target is $S^{2}$. The result was then generalized to general compact surfaces by Qing and Tian [13]. The following result can be found in [13].

PROPOSITION 1. Let $\mathscr{M}$ and $\mathscr{N}$ be closed Riemannian surfaces and let $u_{n} \in$ $W^{1,2}(\mathscr{M}, \mathscr{N})$ be a Palais-Smale sequence for the energy functional such that their tension fields $\tau\left(u_{n}\right)$ are uniformly bounded in $L^{2}(\mathscr{M})$. Then there exist

(i) a finite number of points $x^{i} \in \mathscr{M}, 1 \leq i \leq m<\infty$;

(ii) sequences $\left\{a_{n}^{i}\right\}_{i=1}^{m}$ and $\left\{\lambda_{n}^{i}\right\}_{i=1}^{m}$ satisfying (1.6) and

$$
\lim _{n \rightarrow \infty} a_{n}^{i}=x^{i}, \quad \lim _{n \rightarrow \infty} \lambda_{n}^{i}=0
$$

(iii) a weak limit $u_{\infty}: \mathscr{M} \rightarrow \mathscr{N}$ of $u_{n}$ (or possibly a subsequence) and a finite number of bubbles $\omega_{i}: S^{2} \rightarrow \mathscr{N}$; such that

$$
u_{n}(x)-\sum_{i=1}^{m}\left(\omega_{i}\left(\frac{x-a_{n}^{i}}{\lambda_{n}^{i}}\right)-\omega_{i}(\infty)\right) \rightarrow u_{\infty}(x)
$$

strongly in $W_{\mathrm{loc}}^{1,2}(\mathscr{M}, \mathscr{N})$. Furthermore, the following energy identity holds:

$$
\lim _{n \rightarrow \infty} E_{R}\left(u_{n} ; x_{0}\right)=E_{R}\left(u_{\infty} ; x_{0}\right)+\sum_{i=1}^{m} E_{R}\left(\omega_{i} ; x_{0}\right) \text { for any } R<\infty .
$$

PROOF OF THEOREM 2. To prove Theorem 2, we consider a solution of (1.1) near a singular point $\left(x_{0}, T\right)$. We observe that the energy density is concentrated at the isolated singular points, so that scaling near each singular point is necessary.

If the blow-up time is $T=\infty,(2.8)$ implies we can choose a time sequence $t_{n} \rightarrow \infty$ such that $\tau\left(u\left(\cdot, t_{n}\right)\right)$ is uniformly bounded in $L^{2}(\mathscr{M})$. We select sequences $\left\{\lambda_{n}^{i}\right\}_{i=1}^{m} \rightarrow 0$ and define the scaling $v_{n}(x)=u\left(\lambda_{n} x, t_{n}\right)$. First, by (2.6), note that $E\left(v_{n}\right)=E\left(u\left(\cdot, t_{n}\right)\right)$ is uniformly bounded by $C\left(E_{0}+H_{0}\right)$. Further, $\tau\left(v_{n}\right)=\lambda_{n}^{2} \tau\left(u\left(\cdot, t_{n}\right)\right) \rightarrow 0$. Thus we can check that $E^{\prime}\left(v_{n}\right) \rightarrow 0$ so that $v_{n}(x)$ is a Palais-Smale sequence. We use Proposition 1 on the sequence $v_{n}(x)$ and refer the reader to [12] for details. We 
remark that if we choose $t_{n}$ such that $\tau\left(u\left(\cdot, t_{n}\right)\right) \rightarrow 0$ then the weak limit $u(\cdot, \infty)$ in (1.6) is a harmonic map.

When the blow-up time $T$ is finite, it does not seem possible to choose a time sequence $t_{n} \rightarrow T$ to make $\tau\left(u\left(\cdot, t_{n}\right)\right)$ uniformly bounded in $L^{2}(\mathscr{M})$. However, one can prove the existence of $\lim _{t \rightarrow T} E(u(\cdot, t))$. As in [12], consider the scaling $v_{n}(x)=u\left(\lambda_{n} x+x_{0},-\lambda_{n}^{2} \tau_{n}+t_{n}\right)$ for appropriately chosen sequences $\lambda_{n}, \tau_{n}$ and $t_{n}$ with $\lim _{n \rightarrow \infty} \lambda_{n}=0$ and $\lim _{n \rightarrow \infty} t_{n}=T$. Then $v_{n}(x)$ can be seen to be a Palais-Smale sequence and $\tau\left(v_{n}\right)$ converges to 0 in $L^{2}(\mathscr{M})$ so that again we can apply Proposition 1 to finish the proof.

REMARK 5. Finally, we remark that when the blow-up time is infinite, while the convergence of $\lim _{t \rightarrow \infty} E(u(\cdot, t))$ fails in general, Topping [16] proved that uniform convergence holds when the target manifold is $S^{2}$. He showed that the $L^{2}$-norm of $\tau(u(\cdot, t))$ decreases exponentially in time, and that the weak limit $u(\cdot, \infty)$ and the bubbles $\omega_{i}$ are unique. Furthermore, a counterexample was given to show that these results may not hold when the target manifold is not $S^{2}$. The counterexample is from a two-dimensional domain to $S^{2} \times R^{2}$ on which a warped metric is defined.

\section{Acknowledgement}

Research partially supported by National University of Singapore ARF Grant RP3960688 and the NSF of China Grant 19641001 (third author). Work done while the third author was visiting the National University of Singapore. He gratefully acknowledges the hospitality of the Department of Mathematics, especially Drs Peter Pang and Wang Hongyu.

\section{References}

[1] K. C. Chang and W. Y. Ding, 'A result on the global existence for the heat flows of harmonic maps from $D^{2}$ into $S^{2}$, in: Nemantics: mathematical and physical aspects (eds. J.-M. Coron, J.-M. Ghidaglia and F. Helein) (Kluwer, Dordrecht, 1990) pp. 37-48.

[2] K. C. Chang, W. Y. Ding and R. Ye, 'Finite time blow-up of the heat flow of harmonic maps from surfaces', J. Differential Geom. 36 (1992), 507-515.

[3] Y. M. Chen, 'Blow-up analysis for heat flow of harmonic maps', in: Nemantics: mathematical and physical aspects (eds. J.-M. Coron, J.-M. Ghidaglia and F. Helein) (Kluwer, Dordrecht, 1990) pp. 49-64.

[4] Y. M. Chen and B. Guo, 'Two dimensional Landau-Lifshitz equation', J. Partial Differential Equations 9 (1996), 313-322.

[5] W. Y. Ding and G. Tian, 'Energy identity of a class of approximate harmonic maps from surfaces', Comm. Anal. Geom. 4 (1995), 543-554. 
[6] B. Guo and M. C. Hong, 'The Landau-Lifshitz equations of the ferromagnetic chain and harmonic maps', Calc. Var. Partial Differential Equations 1 (1993), 311-334.

[7] R. S. Hamilton, Harmonic maps of manifolds with boundary, Lecture Notes in Math. 471 (Springer, Berlin, 1975).

[8] M. C. Hong, 'The Landau-Lifshitz equations with the external field-a new extension for the harmonic maps with values in $S^{2}$, Math. Z. 220 (1995), 171-188.

[9] M. C. Hong and L. Lemaire, 'Multiple solutions of the static Landau-Lifshitz equation from $B^{2}$ to $S^{2}$, Math. Z. 220 (1995), 295-306.

[10] L. D. Landau and E. M. Lifshitz, 'On the theory of the dispersion of magnetic permeability in ferromagnetic bodies', Phys. Z. Sowj. 8 (1935), 153; reproduced in Collected works of L. D. Landau (Pergamon Press, New York, 1965) pp. 101-114.

[11] F. H. Lin and C. Y. Wang, 'Energy identity of harmonic maps from surfaces at finite singular time', Calc. Var, Partial Differential Equations 6 (1998), 369-380.

[12] J. Qing, 'On singularities of the heat flow for harmonic maps from surfaces into spheres', Comm. Anal. Geom. 2 (1995), 297-315.

[13] J. Qing and G. Tian, 'Bubbling of the heat flows for harmonic maps from surfaces', Comm. Pure Appl. Math. 50 (1997), 295-310.

[14] C. L. Shen and Q. Zhou, 'The Landau-Lifshitz equations with ferro-magnetic chain', Chinese Ann. Math. Ser. A 18 (1997), 253-262.

[15] M. Struwe, 'On the evolution of harmonic maps of Riemannian surfaces', Comm. Math. Helv. 60 (1985), 558-581.

[16] P. M. Topping, 'Rigidity in the harmonic map heat flow', J. Differential Geom. 45 (1997), 593-610.

[17] Y. D. Wang, 'Heisenberg chain systems from compact manifolds into $S^{2}$, J. Math. Phys. 39 (1998), 363-371.

Department of Mathematics

National University of Singapore

2 Science Drive 2

Republic of Singapore 117543

e-mail: matpyh@nus.edu.sg
Department of Mathematics National University of Singapore 2 Science Drive 2 Republic of Singapore 117543

Current address: Department of Mathematics University of Minnesota Minneapolis MN 55455

USA

e-mail: xiao@math.umn.edu

\section{Department of Mathematics}

East China Normal University

Shanghai 200062

People's Republic of China

e-mail: fzhou@math.ecnu.edu.cn 